\title{
Incidentalomas of the prostate detected by 18-fluoro-2-deoxy-D-glucose positron emission tomography/computed tomography
}

Miles P. Mannas; Taeweon Lee; Maral Pourghiasian; Don Wilson; Peter C. Black Department of Urologic Sciences, University of British Columbia, Vancouver BC, Canada; Vancouver Prostate Centre, Vancouver BC, Canada

Cite as: Can Urol Assoc J 2019 November 29; Epub ahead of print. http://dx.doi.org/10.5489/cuaj.5976

Published online November 29, 2019

$* * *$

\section{Abstract}

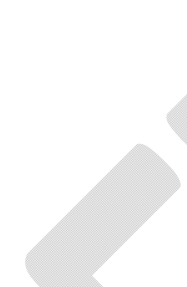

Introduction: Prostate incidentalomas are prostatic lesions suspicious for cancer discovered by imaging patients without a known history of prostatic cancer $(\mathrm{CaP})$ for other reasons. 18-fluoro-2-deoxy-D-glucose positron emission tomography/computed tomography (FDG PET) is used to diagnose, stage and assess response to treatment for numerous cancers, but it is not routinely used for $\mathrm{CaP}$. We aimed to determine the rate of detection of prostate incidentalomas in patients undergoing FDG PET and to evaluate the natural history of these lesions.

Methods: A retrospective review was conducted of all FDG PET scans performed between 2005 and 2017 at a single institution. Patients were selected who had prostatic uptake without a history of prostate cancer. Clinical data were collected from electronic medical records to determine how the prostate incidentalomas were further evaluated and define the rate of malignancy.

Results: A prostate incidentaloma was identified in 309 (1.0\%) of 31019 FDG PET scans performed on men. A prostate-specific antigen (PSA) test was obtained in $40.1 \%$ of patients within six months of prostate incidentaloma detection. Six patients underwent a multiparametric magnetic resonance imaging (MRI) of the prostate, which identified $\mathrm{CaP}$ in one case. Overall, $\mathrm{CaP}$ was diagnosed in 33 cases, representing $10.7 \%$ of the prostate incidentalomas and $0.1 \%$ of the scanned patients. $\mathrm{CaP}$ was intermediate- or high-risk in $27(8.7 \%)$ of the prostate incidentalomas.

Conclusions: Incidental lesions detected in the prostate by FDG PET may represent clinically significant $\mathrm{CaP}$. Referral to a urologist for further evaluation should be considered if the patient is otherwise in reasonable health. 


\section{Introduction}

The shift towards an increase in aerobic glycolysis, known as the Warburg effect, is a well characterized metabolic derangement in cancer. ${ }^{1}$ 18-Fluoro-2-Deoxy-D-Glucose Positron Emission Tomography/Computed Tomography (FDG PET) detects this increase in glycolysis and, as such, is routinely used in the diagnosis and surveillance of many cancers, including melanoma, lung cancer, squamous cell carcinoma of the head and neck, and lymphoma. ${ }^{1,2}$ Prostate cancer $(\mathrm{CaP})$, however, does not routinely experience the Warburg effect and FDG PET is not part of the routine staging of $\mathrm{CaP}^{3-7} \mathrm{An}$ investigation of FDG PET in localized CaP revealed 51.9\% sensitivity and 75.7\% specificity in the context of an elevated prostate specific antigen (PSA). ${ }^{3}$

Incidental focal uptake in any organ on FDG PET that is not directly associated with the disease under investigation is referred to as an incidentaloma. Incidentalomas are commonly observed, occurring in approximately $10-14 \%$ of FDG PET scans. ${ }^{1,2}$ Some of these incidentalomas are located within the prostate in the absence of known $\mathrm{CaP}$. The rate of prostate incidentaloma on FDG PET has been reported in two series as $0.086 \%{ }^{9}$ and $1.4 \% .4$. This elevated prostatic FDG uptake may represent both benign and malignant conditions, including especially $\mathrm{CaP}$, benign prostatic hyperplasia and prostatitis. $^{6}$

To our knowledge, FDG PET prostate incidentalomas have yet to be investigated in a North American population. We aimed to further characterize the etiology, rate of malignancy, aggressiveness of malignancy identified, FDG PET characteristics of identified malignancy, and the adequacy of subsequent investigation once they have been identified. There are also no guidelines to aid in the investigation of these lesions and we sought to develop recommendations for investigation once FDG PET prostate incidentalomas are identified.

\section{Methods}

A retrospective review was conducted of all FDG PET scans performed in men $\geq 18$ years of age between September 2005 and June 2017 at the British Columbia (BC) Cancer Agency. This facility serves the entire population of $\mathrm{BC}$ (over 4.4 million residents in 2016) as the sole PET scanner for the duration of this study period. ${ }^{7}$ This study was approved by the Clinical Research Ethics Board of the BC Cancer Agency (H17-01483).

A search was conducted for all FDG PET reports on male patients that contained the word "prostate". These reports were further narrowed by reviewing the context in the electronic reports. Men with a history of prostate cancer were excluded from further analysis. Full clinical review of the remaining patients was conducted.

A prostate incidentaloma was considered $\mathrm{CaP}$ if this was determined by biopsy (ultrasound guided transrectal (TRUS) biopsy, MRI-TRUS fusion biopsy or transurethral 
resection of prostate) and was considered benign if a biopsy was negative. A prostate incidentaloma was also considered benign if a multiparametric MRI of the prostate was normal, as defined by absence of a PIRADS $\geq 3$ lesion. The prostate incidentalomas were otherwise categorized based on age-standardized PSA gathered from provincial databases that capture results from almost all bloodwork in British Columbia. If the PSA was less than the age-standardized cut-off value (PSA $<2.5 \mathrm{ug} / \mathrm{L}$ for age $<50$ years; PSA $<3.5 \mathrm{ug} / \mathrm{L}$ for age 50-59 years; PSA $<4.5 \mathrm{ug} / \mathrm{L}$ for age 60-69 years; and PSA $<6.5 \mathrm{ug} / \mathrm{L}$ for age $>70$ years), the lesion was considered "likely benign". ${ }^{8}$ Prostate incidentalomas were considered suspicious for $\mathrm{CaP}$ if the PSA was above the age-standardized cut-off level and a biopsy or multiparametric MRI was not performed. The status of the prostate incidentaloma was considered unknown if a PSA could not be located in the databases within 10 years of the FDG PET.

One-way ANOVA and mean statistical testing were performed using Prism 7, version $7.0 \mathrm{e}$.

\section{Results}

In the study period 31,019 FDG PET scans were performed on men over 18 years of age. The number of scans performed per year increased during the study period from 570 per year in 2005 to 9551 per year in 2017, but the rate of detection of prostate incidentalomas remained constant. A prostate incidentaloma was identified in 309 men undergoing FDG PET scanning, representing $1.0 \%$ of all men scanned. The mean age of all men undergoing FDG PET was 63.7 (18-98) years, compared to 70.5 (18-93) years in the patients with a prostate incidentaloma.

Figure 1 shows how the patients were evaluated after FDG PET. The diagnostic categorization of prostate incidentalomas is summarized in Figure 2. A prostate TRUS biopsy was performed in $24(7.8 \%)$ patients within 12 months of detection of the prostate incidentaloma identified on FDG PET, and in 13 (4.2\%) patients beyond 12 months. Prostate cancer was diagnosed in $18(75.0 \%)$ of the early biopsies and $12(92.3 \%)$ of the delayed biopsies (Table 1). The mean PSA of all patients together was $7.3 \mathrm{ug} / \mathrm{L}$ around the time of FDG PET. The mean PSA of patients not undergoing prostate biopsy was $5.9 \mathrm{ug} / \mathrm{L}$, while the PSA of patients undergoing biopsy was $12.1 \mathrm{ug} / \mathrm{L}$. Of patients who went on to be diagnosed with $\mathrm{CaP}$, the mean PSA was $14.9 \mathrm{ug} / \mathrm{L}$. As summarized in Table 2 , of patients who went on to have a prostate biopsy and were diagnosed with CaP, $87.1 \%$ of prostate incidentalomas represented clinically significant $\mathrm{CaP}$ defined as Gleason grade group $\geq 2$. Evaluation of the incidentaloma revealed metastatic $\mathrm{CaP}$ in one patient and 12 others progressed to metastatic $\mathrm{CaP}$ during subsequent follow-up. 
A multiparametric MRI of the prostate was performed in 6 cases. Two patients had no abnormal findings and did not undergo biopsy. A targeted biopsy was performed in 2 patients with a PIRADS 3 lesion and 2 with a PIRADS 4 lesion. These lesions corresponded to the prostate incidentaloma in all cases. One patient with a PIRADS 4 lesion was diagnosed with a Gleason grade group $2 \mathrm{CaP}$. This 68 -year-old patient had a PSA of 1.9 and PSA density of 0.07 . The biopsy was benign in the other cases.

Only 124 (40.1\%) patients underwent PSA testing within 6 months of FDG PET and 147 (47.6\%) patients underwent PSA testing at any point after FDG PET. Based on age-standardized PSA cut-offs, the incidentaloma was deemed likely benign in 117 (37.9\%) patients and suspicious for $\mathrm{CaP}$ in $34(11.0 \%)$ patients who did not undergo prostate biopsy or multiparametric MRI (Figure 1). In 77 (24.9\%) cases no PSA was measured.

The prostate incidentaloma represented a non-prostate malignancy in 35 (11.3\%) patients. In $22(7.1 \%)$ patients it was indicative of direct invasion of rectal carcinoma and in $13(4.2 \%)$ patients it was associated with urothelial carcinoma. Eight of these incidentalomas represented urothelial carcinoma with histologic confirmation of prostatic stromal invasion. Five patients had a prior history of non-muscle invasive bladder cancer and intravesical therapy, but recurrence in the prostate was not confirmed. In no case was the prostate incidentaloma identified to be a metastatic lesion. Furthermore, all CaP cases were adenocarcinoma.

The FDG PET scans were performed to stage different cancers: $34 \%$ lung, 17\% colorectal, 11\% head and neck, 11\% lymphoma, 11\% upper gastrointestinal or hepatobiliary, $4 \%$ melanoma and $6 \%$ for cancer of 10 other origins. Metastatic cancer other than $\mathrm{CaP}$ was present in 137 (44.3\%) patients. Four of these patients underwent prostate biopsy, which was benign in all cases, and $81(59.1 \%)$ were tested for PSA. The mean follow-up of patients after FDG PET was 5.1 years. The mean duration of survival and 5-year survival of patients with a prostate incidentaloma was 3.0 years (range $0-11$ years) and $43.2 \%$, respectively. Three of the 34 patients with a prostate incidentaloma confirmed to be CaP have died of $\mathrm{CaP}$.

One-way ANOVA statistical testing was conducted to investigate whether patient age at time of FDG PET or prostate incidentaloma SUVmax correlated with subsequent diagnosis of CaP. Mean age was not statistically different between likely benign (69.9; 95\% CI 68.11-71.79), suspicious PSA (71.4; 95\% CI 67.45-75.18), CaP on biopsy (71.8; 95\% CI 69.7-74.0) and patients without a PSA measurement (71.4; 95\% CI 68.76-73.84; $\mathrm{p}=0.66$; Figure 3). Similarly, there was no statistically significant difference between the mean SUVmax of likely benign (8.06; 95\% CI 6.93-9.32), suspicious PSA $(8.60 ; 95 \%$ CI 6.85-10.35), biopsy-confirmed $\mathrm{CaP}(9.83 ; 95 \% \mathrm{CI} 7.92-11.74)$ and patients without a PSA measurement $(7.37$; 95\% CI 5.94-8.80; $\mathrm{p}=0.2$; Figure 4$)$. In the reporting of prostate 
incidentalomas, only 204 patients had quantitative measurements of SUVmax, 11 qualitative and 94 had no description other than stating there was abnormal uptake.

\section{Discussion}

We describe a $1.0 \%$ incidence of prostate incidentalomas in men without a history of $\mathrm{CaP}$ undergoing FDG PET imaging for staging of a non-prostate cancer. Since $\mathrm{CaP}$ is the most commonly diagnosed and third leading cause of cancer-related death in Canadian men, ${ }^{9}$ it is not surprising that it can be found incidentally using other imaging modalities. The identification of $\mathrm{CaP}$ relies on digital rectal examination, PSA, and tissue sampling, with multiparametric MRI also taking on a growing role. ${ }^{10,11}$ With increasing use of FDG PET in oncology, it has become more common to encounter patients with prostate incidentalomas, even though $\mathrm{CaP}$ is not typically FDG-avid.

Bertragna et al previously identified equal distribution of incidentalomas between peripheral and transitional zones; however, incidentalomas in the peripheral zone were significantly more often $\mathrm{CaP}^{12}$. Studies have been unable to detect differences in signal intensity (standardized uptake value maximum $\left(\mathrm{SUV}_{\max }\right)$ ) between prostate incidentalomas that are found to be benign or malignant. ${ }^{4,6}$ The rate of $\mathrm{CaP}$ detected in FDG PET prostate incidentalomas is variable in prior reports. ${ }^{1}$ Han et al evaluated $87 \%$ of patients with a prostate incidentaloma and found that $5.4 \%$ represented $\mathrm{CaP} .{ }^{12}$ Bertagna et al evaluated $22.5 \%$ of patients with PSA and biopsy and found CaP in $55.5 \%$ of evaluated cases, or $12.5 \%$ of the overall cohort. ${ }^{12}$ Hwang et al evaluated $12.5 \%$ of cases and found that $12 \%$ of cases contained CaP with Gleason score $\geq 7.6$ Though variant subtypes, of $\mathrm{CaP}$, such as neuroendocrine or ductal $\mathrm{CaP}$, represent $<10 \%$ of all cases, these cancers are more frequently FDG-avid on PET. ${ }^{13,14}$ The aggressive clinical behavior of these tumors is associated with metabolic derangements that allow them to utilize glucose metabolism preferentially. There is evidence that mRNA expression of GLUT1, the first rate limiting step in glucose metabolism, is associated with Gleason score and is upregulated in poorly differentiated castrate resistant $\mathrm{CaP}$, which may correlate to increased FDG uptake. ${ }^{13,14,15}$ However, histologic variants were not detected in our cohort.

In general it is believed that patients likely benefit from treatment of intermediate and high-risk localized CaP if they have a life expectancy of $\geq 10$ years. ${ }^{16}$ Although in our series only $40.1 \%$ of patients underwent PSA testing within 6 months of the prostate incidentaloma identification, and $64.1 \%$ eventually underwent PSA testing or underwent prostate tissue sampling, we are unable to characterize the health status of our patients and are therefore unable to comment on the appropriateness of the delayed investigation of prostate incidentalomas. The competing risk of morbidity and mortality from 
comorbidities and the cancer that the FDG PET was used to stage need to be taken into account.

We have proposed an evaluation algorithm for patients with a prostate incidentaloma on FDG PET without a prior history of CaP (Figure 5). We suggest that all patients should have a serum PSA test and undergo digital rectal examination (DRE), unless they have a severely limited life expectancy (e.g. $<2$ years). Further evaluation with prostate MRI and/or TRUS-guided biopsy would depend on the balance between the patient's general health and the degree of PSA rise or abnormal DRE. We suggest that a patient with a life expectancy greater than 10 years who would be eligible for definitive $\mathrm{CaP}$ treatment should undergo prostate MRI and/or TRUS-guided biopsy to rule out clinically significant prostate cancer. This recommendation is based on the overall experience of detecting aggressive $\mathrm{CaP}$ variants in prostate incidentalomas.

There are significant limitations to this retrospective study. Prostate incidentalomas were identified based on review of FDG PET reports without review of original FDG PET scans. Age standardized PSA was used to classify lesions and exclude $\mathrm{CaP}$; however, patients concluded to have likely benign disease certainly had the possibility to be harboring clinically significant CaP. PSA and follow-up of the cohort was based on EMR review and patients could have had follow-up or lab results not discovered through this review.

\section{Conclusions}

Prostate incidentalomas are an infrequent finding on FDG PET. If the incidentaloma was deemed appropriate for prostate biopsy then typically clinically significant cancer is found. Our results and prior reports in the literature are inadequate to define optimal evaluation of prostate incidentalomas because many patients have undergone no or only very delayed investigation. Depending on other patient variables, this may prove to be the most appropriate management. However, it is important for oncologists to recognize the risk of significant $\mathrm{CaP}$ and to initiate further investigation dependent on patient's underlying prognosis and other factors (age, general health, family history $\mathrm{CaP}$, race). Referral to a urologist should be considered in patients with favorable prognosis to consider MRI and/or prostate biopsy. 


\section{References}

1. Moletta L, Bissoli S, Fantin A, Passuello N, Valmasoni M, Sperti C. PET/CT incidental detection of second tumor in patients investigated for pancreatic neoplasms. BMC Cancer. 2018;18:531.

2. Sone Y, Sobajima A, Kawachi T, Kohara S, Kato K, Naganawa S. Ability of 18fludeoxyglucose positron emission tomography/CT to detect incidental cancer. $\mathrm{Br}$ J Radiol. 2014;87:20140030.

3. Minamimoto R, Uemura H, Sano F, et al. The potential of FDG-PET/CT for detecting prostate cancer in patients with an elevated serum PSA level. Ann Nucl Med. 2011;25:21-7.

4. Bhosale P, Balachandran A, Vikram R, et al. What Is the Clinical Significance of FDG Unexpected Uptake in the Prostate in Patients Undergoing PET/CT for Other Malignancies? Int J Mol Imaging. 2013;2013:476786.

5. Cho SK, Choi JY, Yoo J, et al. Incidental Focal (18)F-FDG Uptake in the Prostate: Clinical Significance and Differential Diagnostic Criteria. Nucl Med Mol Imaging. 2011;45:192-6.

6. Hwang I, Chong A, Jung SI, et al. Is further evaluation needed for incidental focal uptake in the prostate in 18-fluoro-2-deoxyglucose positron emission tomography-computed tomography images? Ann Nucl Med. 2013;27:140-5.

7. Statistics CC. Census Profile, 2016 Census.

8. Oesterling JE. Serum Prostate-Specific Antigen in a Community-Based Population of Healthy Men. JAMA. 1993;270:860-4.

9. C.C. Statistics, Canadian Cancer Society's Advisory Committee On Cancer Statistics., in, Canadian Cancer Society, Canadian Cancer Society, 2015.

10. Force USPST, Grossman DC, Curry SJ, et al. Screening for Prostate Cancer: US Preventive Services Task Force Recommendation Statement. JAMA. 2018;319:1901-13.

11. Kasivisvanathan V, Rannikko AS, Borghi M, et al. MRI-Targeted or Standard Biopsy for Prostate-Cancer Diagnosis. N Engl J Med. 2018;378:1767-77.

12. Bertagna F, Piccardo A, Dib B, et al. Multicentre study of 18F-FDG-PET/CT prostate incidental uptake. Jpn J Radiol. 2015;33:538-46.

13. McEwan LM, Wong D, Yaxley J. Flourodeoxyglucose positron emission tomography scan may be helpful in the case of ductal variant prostate cancer when prostate specific membrane antigen ligand positron emission tomography scan is negative. J Med Imaging Radiat Oncol. 2017;61:503-5.

14. Spratt DE, Gavane S, Tarlinton L, et al. Utility of FDG-PET in clinical neuroendocrine prostate cancer. Prostate. 2014;74:1153-9.

15. Jadvar H. FDG PET in Prostate Cancer. PET Clin. 2009;4:155-61.

16. Heidenreich A, Bastian PJ, Bellmunt J, et al. EAU Guidelines on Prostate Cancer. Part 1: Screening, Diagnosis, and Local Treatment with Curative Intent-Update 2013. European Urology. 2014;65:124-37. 
17. Rendon RA, Mason RJ, Marzouk K, et al. Recommandations de l'Association des urologues du Canada sur le depistage et le diagnostic precoce du cancer de la prostate. Can Urol Assoc J. 2017;11:298-309.

18. Rawla P. Epidemiology of Prostate Cancer. World J Oncol. 2019;10:63-89. 


\section{Figures and Tables}

Fig. 1. Summary of investigations performed to evaluate prostate incidentalomas discovered on FDG PET. FDG PET: 18-fluoro-2-deoxy-D-glucose positron emission tomography/computed tomography; MRI: magnetic resonance imaging; PSA: prostatespecific antigen.

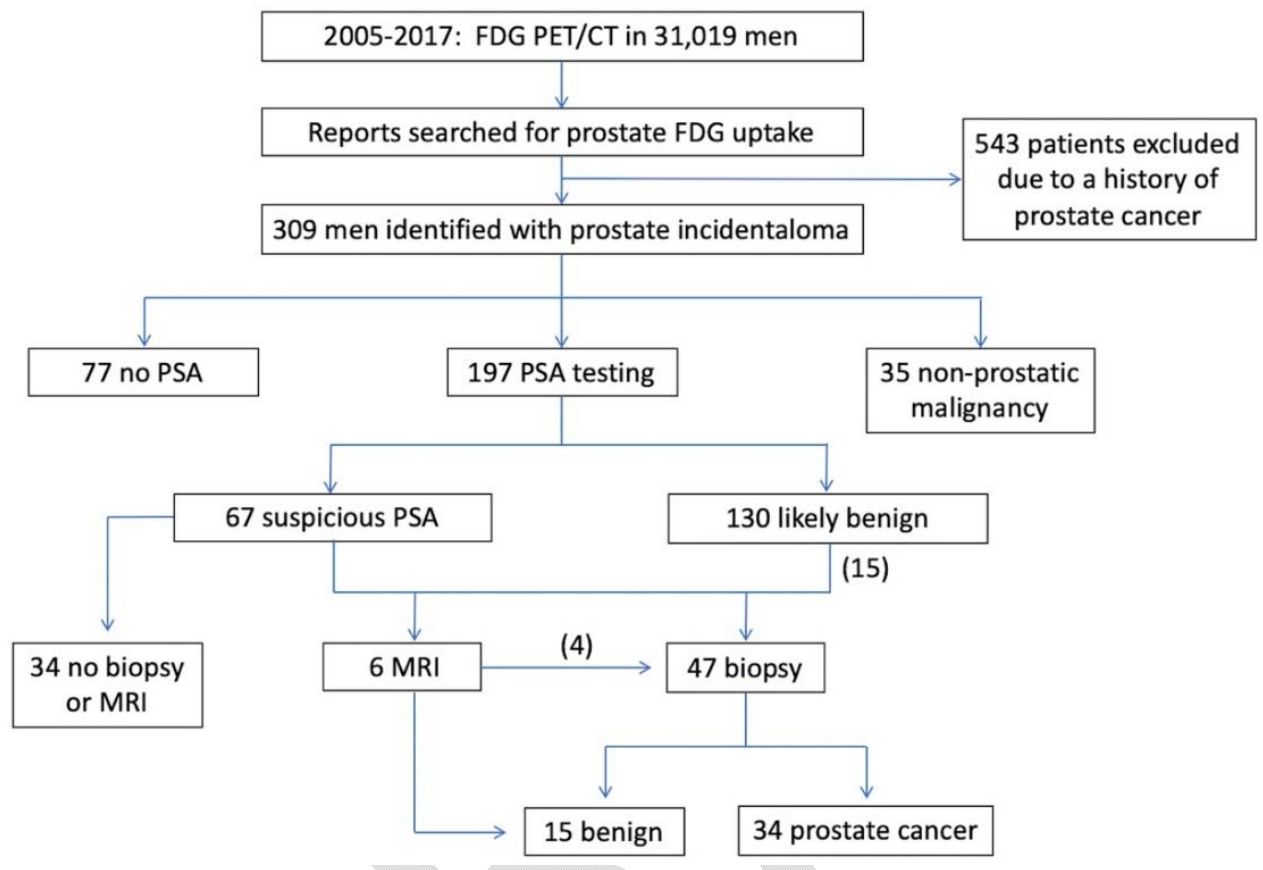

Fig. 2. Classification of prostate incidentalomas based on prostate-specific antigen (PSA) testing and/or prostate biopsy.

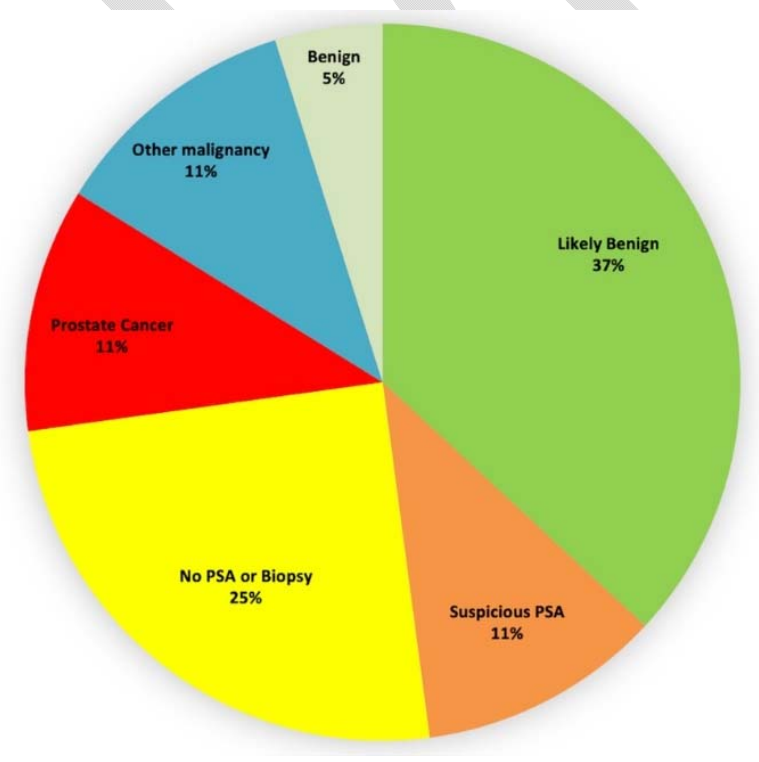


Fig. 3. Patient age at time of prostate incidentaloma detection. PSA: prostate-specific antigen.

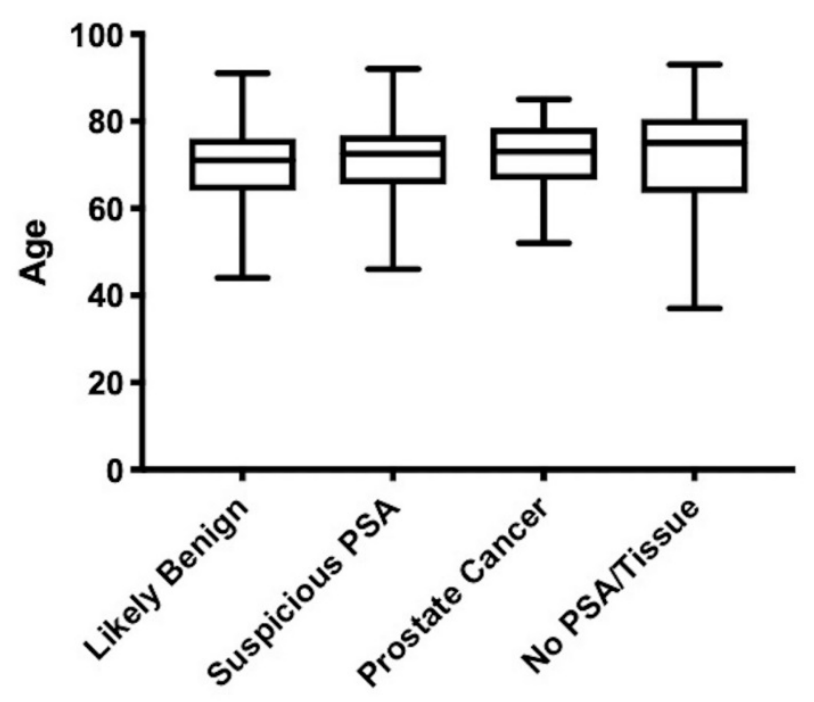

Fig. 4. Prostate incidentaloma $\mathrm{SUV}_{\max }$ PSA: prostate-specific antigen.

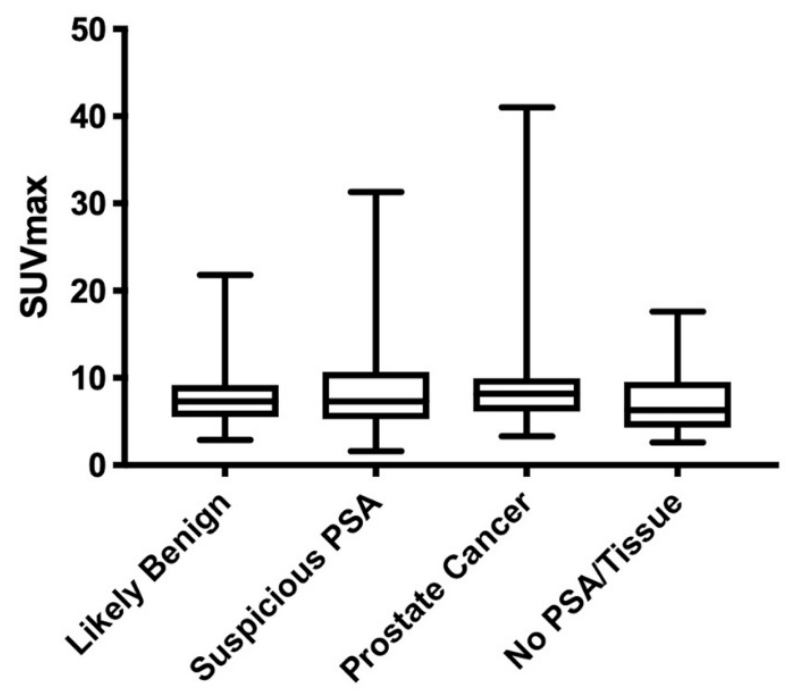


Fig. 5. Proposed algorithm to investigate prostate incidentalomas detected on 18-fluoro2-deoxy-D-glucose positron emission tomography/computed tomography (FDG PET). DRE: digital rectal exam; MRI: magnetic resonance imaging; PSA: prostate-specific antigen; TRUS: transrectal ultrasound.

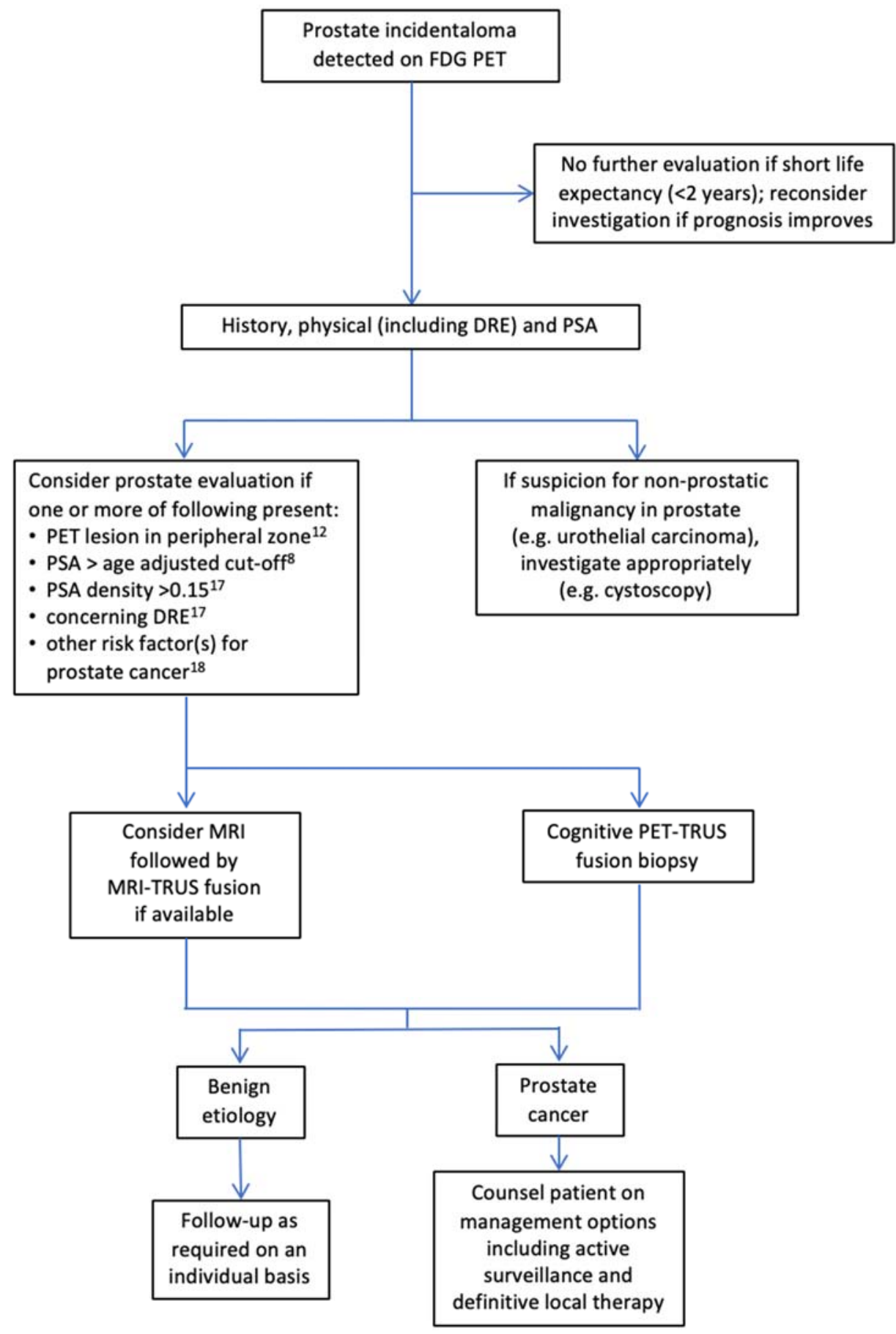




\section{Table 1. Time to diagnosis of prostate cancer after detection of prostate incidentaloma on FDG PET}

\begin{tabular}{|l|c|c|}
\hline $\begin{array}{l}\text { Time to } \\
\text { diagnosis }\end{array}$ & $\begin{array}{c}\text { Number of } \\
\text { patients }\end{array}$ & Gleason grade group \\
\hline$<1$ year & 20 & $1-5$ \\
\hline $1-2$ years & 7 & $1-3$ \\
\hline $2-3$ years & 2 & 5 \\
\hline 3-4 years & 1 & 3 \\
\hline $4-5$ years & 3 & 2 and 5 \\
\hline $5-6$ years & 1 & 5 \\
\hline
\end{tabular}

FDG PET: 18-fluoro-2-deoxy-D-glucose positron emission tomography/computed tomography.

\begin{tabular}{|l|c|}
\hline \multicolumn{2}{|l|}{ Table 2. Prostate cancer diagnosed after FDG } \\
PET & Number of patients \\
\hline Gleason grade group & 5 \\
\hline 1 & 6 \\
\hline 2 & 6 \\
\hline 3 & 3 \\
\hline 4 & 12 \\
\hline 5 & 1 \\
\hline De novo metastatic disease & \\
\hline
\end{tabular}

FDG PET: 18-fluoro-2-deoxy-D-glucose positron emission tomography/computed tomography. 